\title{
Strong Magnetoresistance Induced by Long-Range Disorder
}

\author{
A. D. Mirlin, ${ }^{1, *}$ J. Wilke, ${ }^{1}$ F. Evers, ${ }^{1}$ D. G. Polyakov,${ }^{2, \dagger}$ and P. Wölfle $e^{1,2}$ \\ ${ }^{1}$ Institut für Theorie der Kondensierten Materie, Universität Karlsruhe, 76128 Karlsruhe, Germany \\ ${ }^{2}$ Institut für Nanotechnologie, Forschungszentrum Karlsruhe, 76021 Karlsruhe, Germany
}

(Received 25 May 1999)

\begin{abstract}
We calculate the semiclassical magnetoresistivity $\rho_{x x}(B)$ of noninteracting fermions in two dimensions moving in a weak and smoothly varying random potential or random magnetic field. We demonstrate that in a broad range of magnetic fields the non-Markovian character of the transport leads to a strong positive magnetoresistance. The effect is especially pronounced in the case of a random magnetic field where $\rho_{x x}(B)$ becomes parametrically much larger than its $B=0$ value.
\end{abstract}

PACS numbers: 73.40.-c, 05.60.-k, 73.50.Jt

The magnetoresistance (MR) is one of the most frequently studied characteristics of the two-dimensional electron gas (2DEG). When the effect of disorder is described by a collision integral within the semiclassical Boltzmann equation approach, the resistivity tensor $\hat{\rho}(B)$ for an isotropic system has the Drude form

$$
\hat{\rho}(B)=\frac{m}{e^{2} n}\left(\begin{array}{cc}
\tau^{-1} & \omega_{c} \\
-\omega_{c} & \tau^{-1}
\end{array}\right)
$$

where $n$ is the carrier density, $m$ the effective mass, $\omega_{c}=e B / m c$ the cyclotron frequency, and $\tau$ the transport scattering time. In particular, the longitudinal resistivity $\rho_{x x}$ is independent of the magnetic field $B, \rho_{x x}(B)=$ $\rho_{0} \equiv m / e^{2} n \tau$, irrespective of the form of the impurity collision integral. This result is solely determined by the Markovian character of the transport assumed in the Boltzmann equation description.

Deviations from the constant $\rho_{x x}(B)$ are conventionally termed a positive/negative MR, depending on the sign of the deviation. The negative MR [1] induced by the suppression of the quantum interference correction by the magnetic field is a famous manifestation of weak localization. Another source of negative MR is the AltshulerAronov correction to the conductivity due to enhancement of the electron-electron interaction by the diffusive motion of particles [1]. Both these effects are of quantum nature and lead to a correction of order $e^{2} / h$ to the conductivity $\sigma_{x x}$, and thus to a small correction to $\rho_{x x}$.

However, as we will show, already at the classical level there exists a nontrivial MR which can be much stronger than the quantum one, if the correlation length $d$ of disorder is sufficiently large, $k_{F} d \gg 1$ (where $k_{F}$ is the Fermi wave vector). This is due to memory effects which are neglected in the collision integral description of disorder.

Transport properties of the 2DEG in a smooth random potential (RP) $V(\mathbf{r})$ are of particular interest, since in currently fabricated high-mobility semiconductor heterostructures the disorder has long-range character. The high mobility of these samples is achieved by placing the charged donor ions in a layer separated by a large distance $d\left(k_{F} d \sim 10\right)$ from the 2DEG plane. Assuming the positions of these impurities to be statistically distributed with a sheet density $n_{i}$, the correlation function $W_{V}(\mathbf{r}-$ $\left.\mathbf{r}^{\prime}\right)=\left\langle V(\mathbf{r}) V\left(\mathbf{r}^{\prime}\right)\right\rangle$ is given in momentum space by

$$
\tilde{W}_{V}(q)=\left(\pi \hbar^{2} / m\right)^{2} n_{i} e^{-2 q d} .
$$

A new type of transport problem occurs in these systems when a large magnetic field $B \simeq B_{1 / 2}=2(h c / e) n$ is applied such that the lowest Landau level is approximately half filled. The metallic state then observed has been described [2] in terms of composite fermions (CF's) moving in a weak effective field $\bar{B}=B-B_{1 / 2}$. The CF's are scattered by an impurity-induced random magnetic field (RMF) $\delta B(\mathbf{r})$ characterized by the correlation function $W_{B}\left(\mathbf{r}-\mathbf{r}^{\prime}\right)=\left\langle\delta B(\mathbf{r}) \delta B\left(\mathbf{r}^{\prime}\right)\right\rangle$ with Fourier components

$$
\tilde{W}_{B}(q)=(2 h c / e)^{2} n_{i} e^{-2 q d} .
$$

While the above RMF is fictitious, a real long-range correlated RMF can also be realized in semiconductor heterostructures by attaching superconducting $[3,4]$ or ferromagnetic $[5,6]$ overlayers. We will study below the case of a weak RP or RMF, which means that $l \gg d$, where $l$ is the mean free path in zero average field $\bar{B}=0$. Let us stress that we consider a situation with only the smooth disorder (RP or RMF) present. This should be contrasted with the starting point of [7], where the resistivity was assumed to be dominated by a white-noise RP while a weak long-range RMF was considered as a small perturbation.

As one manifestation of the strongly non-Markovian transport, it has been shown recently [8-10] that in a sufficiently strong $\bar{B}$ the MR drops exponentially with $\bar{B}$ because of a "classical localization" effect caused by adiabacity of the motion. This holds true for the motion both in a RP [8] and in a RMF [9,10]. The condition of this adiabatic regime is $\omega_{c} \tau \gg(l / d)^{2 / 3}$.

In this paper we study the region of smaller magnetic fields in which different non-Markovian processes become important. We find that the exponential falloff of $\rho_{x x}$ is preceded by a strong positive MR. The effect is especially pronounced in the case of the RMF, where the increase of $\rho_{x x}$ is much larger (in the weak disorder limit) than its zero- $\bar{B}$ value $\rho_{0}=m / e^{2} n \tau$. 
We first outline the physics of the effect on a qualitative level. The zero- $\bar{B}$ transport scattering rates in the RP and $\mathrm{RMF}$ are given by [11]

$$
\begin{gathered}
\frac{1}{\tau}=\frac{1}{2 \pi m^{2} v_{F}^{3}} \int_{0}^{\infty} d q q^{2} \tilde{W}_{V}(q) \quad(\mathrm{RP}) \\
\frac{1}{\tau}=\left(\frac{e}{m c}\right)^{2} \frac{1}{2 \pi v_{F}} \int_{0}^{\infty} d q \tilde{W}_{B}(q) \quad(\mathrm{RMF}) .
\end{gathered}
$$

Let us now discuss the nature of the particle trajectories. The change $d \phi$ of the polar angle of the particle velocity in the time interval $d t$ is given by

$$
\begin{gathered}
d \phi \simeq\left(m v_{F}\right)^{-1} n_{i} \epsilon_{i j} \partial_{j} V(\mathbf{r}) d t \quad(\mathrm{RP}) ; \\
d \phi=(e / m c) \delta B(\mathbf{r}) d t \quad(\mathrm{RMF}),
\end{gathered}
$$

where $\mathbf{n}=\mathbf{v} /|\mathbf{v}|$ is the unit vector in the direction of the velocity. Taking into account that the trajectory within the correlation domain of the size $\sim d$ is almost a straight line, one gets (4) and (5). One source of the MR is the bending of the trajectory within the correlation domain by the magnetic field $\bar{B}$. This leads to a small negative MR, of the relative magnitude $\sim\left(d / R_{c}\right)^{2} \ll 1[9,12]$. There exists, however, a much stronger effect related to returns of the particle to spatial regions close to the starting point. Under the condition $\omega_{c} \tau \gg 1$ the particle trajectory is a sequence of slightly distorted cyclotron circles. The center of the orbit is shifted by a random vector $\boldsymbol{\delta}$ after one cyclotron revolution, with $\langle\boldsymbol{\delta}\rangle=0,\left\langle\delta_{x}^{2}\right\rangle=\left\langle\delta_{y}^{2}\right\rangle=$ $2 \pi l^{2} /\left(\omega_{c} \tau\right)^{3}$. The correlation of the elementary scattering processes [Eqs. (6) and (7)] occurs in the vicinity of the points where the cyclotron orbits intersect each other, which leads to a correction to the resistivity

$$
\begin{aligned}
& \left(\Delta \rho_{x x} / \rho_{0}\right)_{1} \sim\left[d /\left\langle\boldsymbol{\delta}^{2}\right\rangle^{1 / 2}\right]^{3} \quad(\mathrm{RP}) \\
& \left(\Delta \rho_{x x} / \rho_{0}\right)_{1} \sim d /\left\langle\boldsymbol{\delta}^{2}\right\rangle^{1 / 2} \quad(\mathrm{RMF}) .
\end{aligned}
$$

The same is valid for $t \simeq 2 \pi n / \omega_{c}$ (the $n$th cyclotron revolution, $n=2,3, \ldots$ ), with $\left\langle\boldsymbol{\delta}^{2}\right\rangle$ multiplied by $n$. For the RP case the corresponding sum over $n$ converges, $\sum_{n=1}^{\infty} n^{-3 / 2}=\zeta(3 / 2)$, leading simply to the renormalization of a numerical factor in (8). In fact, in this case the above consideration can be made fully quantitative, and the result agrees with what we will find below from the Liouville equation (10). The corresponding positive MR, $\Delta \rho_{x x} / \rho_{0} \sim(d / l)^{3}\left(\omega_{c} \tau\right)^{9 / 2}$, becomes of order unity at the upper bound of the considered range of the magnetic fields, $\omega_{c} \tau \lesssim(l / d)^{2 / 3}$. Here the system enters the adiabatic regime.

In the RMF case the sum over $n$ is of the form $\sum n^{-1 / 2}$ and is thus determined by the upper cutoff, which is $n \sim$ $\omega_{c} \tau$. The resulting correction $\Delta \rho_{x x} / \rho_{0} \sim(d / l)\left(\omega_{c} \tau\right)^{2}$ reaches a value of order unity at $\omega_{c} \tau \sim(l / d)^{1 / 2}$, i.e., far from the adiabatic regime. At larger magnetic fields, where $\Delta \rho_{x x} / \rho_{0} \gg 1$, a self-consistent treatment is needed (see below).

We describe now a formalism which allows us to calculate the MR more systematically (see also [7]). We consider first the RMF case. The starting point is the Liouville equation

$$
\begin{gathered}
\left(L_{0}+\delta L\right) g(\omega, \mathbf{r}, \phi)=\cos \left(\phi-\phi_{E}\right) \\
L_{0}=-i \omega+v_{F} \mathbf{n} \nabla+\frac{e}{m c} \bar{B} \frac{\partial}{\partial \phi} \\
\delta L=\frac{e}{m c} \delta B(\mathbf{r}) \frac{\partial}{\partial \phi}
\end{gathered}
$$

for the deviation $\delta f(\omega, \mathbf{r}, \phi)=e E v_{F} \frac{\partial f_{0}}{\partial \epsilon} g(\omega, \mathbf{r}, \phi)$ from the equilibrium distribution function $f_{0}=\theta\left(\epsilon_{F}-\epsilon\right)$. Here $\mathbf{E}=E\left(\cos \phi_{E}, \sin \phi_{E}\right)$ is the electric field and $\mathbf{n}=$ $(\cos \phi, \sin \phi)$ the unit vector determining the velocity direction. The current density is given by $\mathbf{j}=-e \times$ $\int \frac{d^{2} p}{(2 \pi \hbar)^{2}} \mathbf{v} \delta f$, which yields the conductivity tensor

$\hat{\sigma}=e^{2} v_{F}^{2} N_{F} \int \frac{d \phi}{2 \pi}\left\langle\left(\begin{array}{c}\cos \phi \\ \sin \phi\end{array}\right)\left(L_{0}+\delta L\right)^{-1}\left(\begin{array}{c}\cos \phi \\ \sin \phi\end{array}\right)^{T}\right\rangle$,

where $N_{F}$ is the density of states and the angular brackets denote the averaging over configurations of the RMF $\delta B(\mathbf{r})$ with the correlation function (3).

Expanding (11) in $\delta L$, averaging over the disorder and resumming the series (in the same way as it is done for a quantum-mechanical Green's function), one arrives at

$$
\begin{aligned}
\hat{\rho}=\hat{\sigma}^{-1} & =\frac{2}{e^{2} v_{F}^{2} N_{F}}\left(\hat{L}_{0}+\hat{M}\right) ; \\
\hat{L}_{0} & =\left(\begin{array}{cc}
-i \omega & \omega_{c} \\
-\omega_{c} & -i \omega
\end{array}\right),
\end{aligned}
$$

where $M$ is the "self-energy" and the $2 \times 2$ matrix $\hat{M}$ is defined by

$$
\hat{M}=\int \frac{d \phi}{\pi}\left(\begin{array}{c}
\cos \phi \\
\sin \phi
\end{array}\right) M\left(\begin{array}{c}
\cos \phi \\
\sin \phi
\end{array}\right)^{T} .
$$

In zero $\bar{B}$ the self-energy $M$ is given in the leading approximation by the first term of the perturbative expansion, $M=-\left\langle\delta L L_{0}^{-1} \delta L\right\rangle$, yielding

$$
\begin{aligned}
M_{x x}= & -2 i\left(\frac{e}{m c}\right)^{2} \int \frac{d^{2} q}{(2 \pi)^{2}} \frac{d \phi}{2 \pi} \\
& \times \sin \phi \frac{\tilde{W}_{B}(q)}{v_{F} q \cos \left(\phi-\phi_{q}\right)-\omega} \sin \phi,
\end{aligned}
$$

where $\phi_{q}$ is the polar angle of the momentum q. Taking into account that $\omega$ should have an infinitesimal positive imaginary part $(\omega \rightarrow \omega+i 0)$ and considering the limit $\omega \rightarrow 0$, we get $M_{x x}=1 / \tau$ with $1 / \tau$ given by (5). Equation (12) reproduces then the Drude resistivity, as expected.

Now we calculate the $\bar{B}$-dependent correction to (14) determined by the return processes described above. For this purpose, we have to replace the free propagator $L_{0}^{-1}=$ $\left[-i \omega+i v_{F} q \cos \left(\phi-\phi_{q}\right)\right]^{-1}$ entering (14) by the one 
describing the motion in the magnetic field in the presence of disorder. At small $q$ this would be simply the diffusion propagator; however, since we are interested in the shortscale physics, $q \gtrsim R_{c}^{-1}$, the diffusion approximation is not appropriate. Using the fact that the particle is scattered only by a small angle within the correlation length $d$, we can approximate the motion by a Fokker-Planck equation corresponding to the diffusion in momentum space:

$$
\begin{aligned}
\Delta M_{x x}= & 2\left(\frac{e}{m c}\right)^{2} \int \frac{d^{2} q}{(2 \pi)^{2}} \tilde{W}_{B}(q) \\
& \times \int \frac{d \phi}{2 \pi} \sin \phi g_{\mathrm{D}}(\omega, \mathbf{q}, \phi),
\end{aligned}
$$

where $g_{\mathrm{D}}$ is the solution of

$$
\begin{aligned}
& {\left[-i \omega+i v q \cos \left(\phi-\phi_{q}\right)+\omega_{c} \frac{\partial}{\partial \phi}-\right.} \\
& \left.\frac{1}{\tau} \frac{\partial^{2}}{\partial \phi^{2}}\right] g_{\mathrm{D}}(\omega, \mathbf{q}, \phi)=\sin \phi .
\end{aligned}
$$

Now we make use of the fact that the solution of (16) determines the spatial dispersion of the conductivity in the situation of small-angle scattering $[13,14]$ :

$$
\sigma_{y y}(\omega, \mathbf{q})=e^{2} v_{F}^{2} N_{F} \int \frac{d \phi}{2 \pi} \sin \phi g_{\mathrm{D}}(\omega, \mathbf{q}, \phi) .
$$

At $\omega=0$ one has $\sigma_{y y}(\mathbf{q} \| \hat{\mathbf{y}})=0$ [13]. For $\mathbf{q} \| \hat{\mathbf{x}}$ we get, using the solution of (16) at $\omega_{c} \tau \gg 1$ [14],

$$
\begin{aligned}
\sigma_{y y}(0, \mathbf{q}) & \simeq \frac{2 \sigma_{0}}{\omega_{c} \tau} \int_{0}^{2 \pi} \frac{d \phi}{2 \pi} \int_{-\infty}^{\phi} d \phi^{\prime} \sin \phi \sin \phi^{\prime} e^{-K\left(\phi, \phi^{\prime}\right)} \\
& \simeq 4 \sigma_{0} J_{1}^{2}\left(q R_{c}\right) /\left(q R_{c}\right)^{2},
\end{aligned}
$$

where $\quad K\left(\phi, \phi^{\prime}\right)=i q R_{c}\left(\sin \phi-\sin \phi^{\prime}\right)-\left[\left(q R_{c}\right)^{2} /\right.$ $\left.2 \omega_{c} \tau\right]\left(\phi-\phi^{\prime}\right)$ and $\sigma_{0}=\rho_{0}^{-1}$. This determines the integrand of (15) for general orientation of $\mathbf{q}$, with the result for $\omega=0$

$$
\begin{aligned}
\Delta M_{x x} & =\left(\frac{e}{m c}\right)^{2} \frac{\tau}{\pi R_{c}^{2}} \int_{0}^{\infty} \frac{d q}{q} J_{1}^{2}\left(q R_{c}\right) \tilde{W}_{B}(q) \\
& \simeq\left(\frac{e}{m c}\right)^{2} \frac{\tau}{2 \pi R_{c}^{2}} \tilde{W}_{B}(0) .
\end{aligned}
$$

In agreement with the above qualitative picture, the main contribution to the integral comes from $q \sim R_{c}^{-1}$. Therefore, we neglected the $q$ dependence of $\tilde{W}_{B}(q)$ in the second line of (19), in view of $d \ll R_{c}$. The MR is thus positive and quadratic in $\bar{B}$,

$$
\Delta \rho_{x x} / \rho_{0}=\left(\bar{B} / B_{0}\right)^{2} \equiv 4 \alpha^{2}\left(\omega_{c} \tau\right)^{2} \equiv 2(d / l)\left(\omega_{c} \tau\right)^{2},
$$

where $B_{0}=\left\langle\delta B^{2}(\mathbf{r})\right\rangle^{1 / 2} \equiv W_{B}^{1 / 2}(0)$ is the amplitude of the RMF fluctuations and $\alpha \ll 1$ is the parameter characterizing the RMF strength $[9,10], \alpha=d \omega_{c}^{(0)} / v_{F}$ with $\omega_{c}^{(0)}=e B_{0} / m c$. Equation (20) is valid for $\bar{B} \ll B_{0}$, whereas the adiabatic regime begins at $\bar{B} \sim B_{0} \alpha^{-1 / 3}$. In the intermediate range, $B_{0} \lesssim \bar{B} \lesssim B_{0} \alpha^{-1 / 3}$, the positive MR gets large, $\Delta \rho_{x x} / \rho_{0} \gg 1$. In this region, Eq. (19) should be treated self-consistently, i.e., $\tau$ in the righthand side should be understood as a renormalized scattering time, $\tau^{-1}=\tau_{0}^{-1}+\Delta M_{x x}$. The result for $\bar{B} \gg B_{0}$ is $\rho_{x x} / \rho_{0}=\bar{B} / B_{0}$, or, in the form valid both below and above $B_{0}$,

$$
\rho_{x x} / \rho_{0}=1 / 2+\left[1 / 4+\left(\bar{B} / B_{0}\right)^{2}\right]^{1 / 2},
$$

which is our main result for the case of RMF. At $\bar{B} / B_{0} \sim$ $\alpha^{-1 / 3}$ the resistivity reaches its maximum $\rho_{x x} \sim$ $\alpha^{-1 / 3} \rho_{0}$; in still higher fields $\rho_{x x}$ drops rapidly due to the adiabatic character of motion.

We turn now to the RP scattering. The operator $\delta L$ in (10) then has the form

$$
\delta L=\delta v(\mathbf{r}) \mathbf{n} \nabla+[\nabla \delta v(\mathbf{r})] \mathbf{n}_{\perp} \frac{\partial}{\partial \phi},
$$

where $\delta v(\mathbf{r})=V(\mathbf{r}) / p_{F}$ is the spatial variation of the Fermi velocity and $\mathbf{n}_{\perp}=\hat{\mathbf{z}} \times \mathbf{n}=(-\sin \phi, \cos \phi)$. At zero $\bar{B}$ we get, instead of (14),

$$
\begin{aligned}
M_{x x}= & -\frac{2 i}{p_{F}^{2}} \int \frac{d^{2} q}{(2 \pi)^{2}} \frac{d \phi}{2 \pi} \sin \phi \sin \left(\phi-\phi_{q}\right) \\
& \times \frac{q^{2} \tilde{W}_{V}(q)}{v_{F} q \cos \left(\phi-\phi_{q}\right)-\omega} \sin \phi \sin \left(\phi-\phi_{q}\right),
\end{aligned}
$$

reproducing the result (4). To calculate the MR, we replace, as in the RMF case, the free propagator in (23) by $L_{\mathrm{D}}^{-1}$, where $L_{\mathrm{D}}$ is the operator in the square brackets in (16). Solving again the equation $L_{\mathrm{D}} g_{\mathrm{D}}(\omega, \mathbf{q}, \phi)=$ $\sin \phi \sin \left(\phi-\phi_{q}\right)$ at $\omega_{c} \tau \gg 1$, we find after some algebraic manipulations

$$
\begin{aligned}
\Delta M_{x x} \simeq & \frac{1}{2 \pi p_{F}^{2} v_{F}} \int_{0}^{\infty} d q q^{2} \tilde{W}_{V}(q) \\
& \times\left[\operatorname{coth} \frac{\pi\left(q R_{c}\right)^{2}}{2 \omega_{c} \tau}-1\right] .
\end{aligned}
$$

In contrast to (19), the integral is determined by the region of momenta $q^{2} \sim \omega_{c} \tau / R_{c}^{2} \sim\left\langle\boldsymbol{\delta}^{2}\right\rangle^{-1}$, as might be expected from the above qualitative consideration. Since $d \ll\left\langle\boldsymbol{\delta}^{2}\right\rangle^{1 / 2}$, we can again neglect the $q$ dependence of $\tilde{W}_{V}(q)$, which yields for $\omega_{c} \tau \ll(l / d)^{2 / 3}$

$$
\begin{aligned}
\frac{\Delta \rho_{x x}}{\rho_{0}} & =\frac{\zeta(3 / 2)}{4 \pi^{2}} \frac{\tau \tilde{W}_{V}(0)}{p_{F}^{2} v_{F} l^{3}}\left(\omega_{c} \tau\right)^{9 / 2} \\
& =\frac{2 \zeta(3 / 2)}{\pi}\left(\frac{d}{l}\right)^{3}\left(\omega_{c} \tau\right)^{9 / 2}
\end{aligned}
$$

We have performed numerical simulations of the MR for both types of disorder (RMF and RP). In Fig. 1 the results for the RMF are shown, for three different strengths of the disorder $(\alpha=0.2,0.083,0.0138)$. At $\alpha \ll 1$, the theoretical prediction of the strong positive MR (21) 


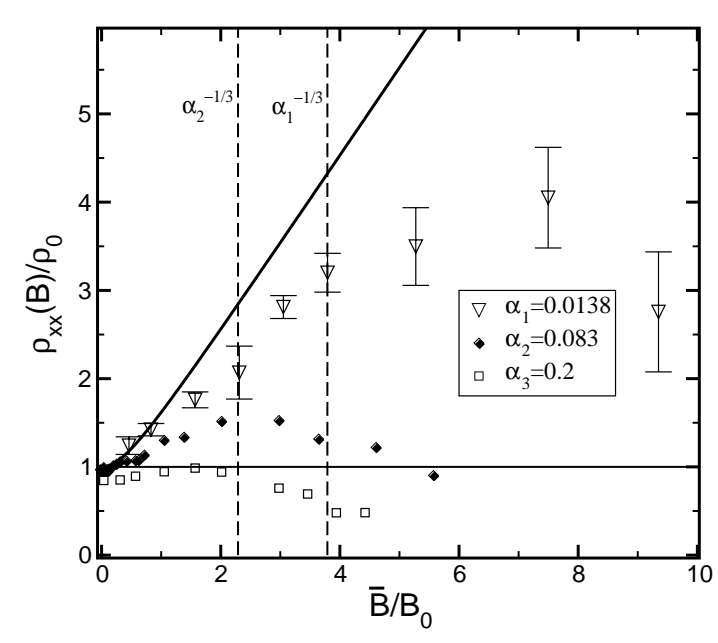

FIG. 1. Magnetoresistivity [normalized by the Drude value $\rho_{0}=m / e^{2} n \tau$ with $1 / \tau$ given by (5)] in random magnetic field from the numerical simulations for three different strengths of the disorder; the full line corresponds to Eq. (21).

crossing over to the negative one at $\bar{B} \sim B_{0} \alpha^{-1 / 3}$ is fully confirmed by the data. At moderately small $\alpha$ ( $\alpha=0.2$ in Fig. 1) the positive MR still exists, but becomes weak; this is the region of $\alpha$ relevant to the composite-fermion description of the vicinity of half filling of the lowest Landau level $(\nu=1 / 2)$. The numerically calculated MR for $\alpha \sim 0.2-0.3$ agrees well [10] with the experimental data [15] around $\nu=1 / 2$. At large $\alpha \geq 0.5$ the region of positive MR disappears, and $\rho_{x x}$ drops monotonously with $\bar{B}[10]$.

A pronounced positive MR induced by a real long-range RMF was also observed in [5]. While a direct application of our results is not possible in this case, because of the presence of a strong RP with a much shorter correlation length, we believe that the physics of the effect is essentially the same.

The numerically found MR for the RP case (Fig. 2) shows good agreement with the theoretical result (24) up

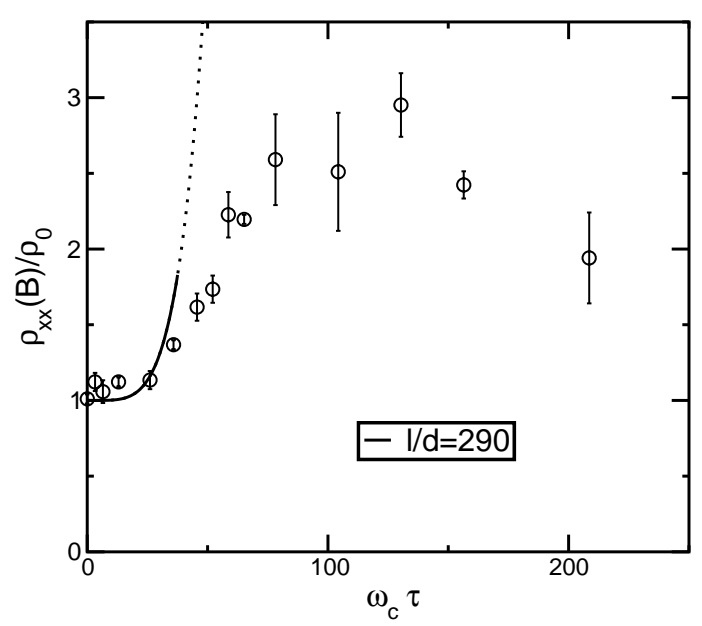

FIG. 2. Magnetoresistivity in a random potential from computer simulations in comparison with Eq. (24). to $\Delta \rho_{x x} / \rho_{0} \sim 1$. At larger $\bar{B}, \rho_{x x}$ deviates from (24) and starts to decrease, as expected.

In conclusion, we have demonstrated that the $2 \mathrm{D}$ fermion gas shows for weak long-range correlated disorder a strong positive MR in moderately strong magnetic fields $1 \ll \omega_{c} \tau \lesssim(l / d)^{2 / 3}$, due to the non-Markovian character of transport. The effect is especially pronounced in the case of the RMF. Our findings explain, in particular, the positive MR of composite fermions observed experimentally around $\nu=1 / 2$.

This work was supported by the SFB 195 der Deutschen Forschungsgemeinschaft and by the INTAS Grant No. 97-1342.

*Also at Petersburg Nuclear Physics Institute, 188350 St. Petersburg, Russia.

${ }^{\dagger}$ Also at A. F. Ioffe Physico-Technical Institute, 194021 St. Petersburg, Russia.

[1] See, e.g., P. A. Lee and T. V. Ramakrishnan, Rev. Mod. Phys. 57, 287 (1985); B. L. Altshuler and A. G. Aronov, in Electron-Electron Interactions in Disordered Systems, edited by A.L. Efros and M. Pollak (North-Holland, Amsterdam, 1985).

[2] B. I. Halperin, P. A. Lee, and N. Read, Phys. Rev. B 47, 7312 (1993).

[3] S. J. Bending, K. von Klitzing, and K. Ploog, Phys. Rev. Lett. 65, 1060 (1990).

[4] A. Geim, S. Bending, and I. Grigorieva, Phys. Rev. Lett. 69, 2252 (1992); A. Geim, S. Bending, I. Grigorieva, and M. G. Blamire, Phys. Rev. B 49, 5749 (1994).

[5] P.D. Ye, D. Weiss, G. Lütjering, R. R. Gerhardts, K. von Klitzing, K. Eberl, H. Nickel, and G. Weimann, in Proceedings of the 23rd International Conference on The Physics of Semiconductors (World Scientific, Singapore, 1996), p. 1529.

[6] F. B. Mancoff, R. M. Clarke, C. M. Marcus, S. C. Zhang, K. Campman, and A.C. Gossard, Phys. Rev. B 51, 13269 (1995); L. Zielinski, K. Chaltikian, K. Birnbaum, C. M. Marcus, K. Campman, and A. C. Gossard, Europhys. Lett. 42, 73 (1998).

[7] P. Hedegard and A. Smith, Phys. Rev. B 51, 10869 (1995).

[8] M. M. Fogler, A. Yu. Dobin, V.I. Perel, and B.I. Shklovskii, Phys. Rev. B 56, 6823 (1997).

[9] A. D. Mirlin, D. G. Polyakov, and P. Wölfle, Phys. Rev. Lett. 80, 2429 (1998).

[10] F. Evers, A.D. Mirlin, D. G. Polyakov, and P. Wölfle, cond-mat/9901070 [Phys. Rev. B (to be published)].

[11] A. D. Mirlin, E. Altshuler, and P. Wölfle, Ann. Phys. (Leipzig) 5, 281 (1996).

[12] D. V. Khveshchenko, Phys. Rev. Lett. 77, 1817 (1996).

[13] A. D. Mirlin and P. Wölfle, Phys. Rev. Lett. 78, 3717 (1997).

[14] A.D. Mirlin and P. Wölfle, Phys. Rev. B 58, 12986 (1998).

[15] See, e.g., J.H. Smet, in Composite Fermions, edited by O. Heinonen (World Scientific, Singapore, 1998), Fig. 1. 